\title{
Wood-Concrete and Wood-Wood Mixed Beams: Rational Basis for Selecting Connections ${ }^{1}$
}

\author{
José L. Fernández-Cabo, Ph.D. , Jorge Fernández-Lavandera ; and José M. Ávila-Jalvo, Ph.D.
}

\begin{abstract}
Many studies have approached the problem of mixed beams with a slip at the connection. An analysis of the problem has been carried out globally and Eurocode-5 solves the problem for the use of simple supported beams or similar structures in a precise way and with a simple formulation. Other methods to solve the structural analysis of this issue include matrix as well as several finite-element procedures. Nevertheless, the design process for selecting the connection is now iterative and the variables of the problem can be presented in a more condensed and useful way. The problems of strength and stiffness in this research have been separated. Variables in a powerful nondimensional way and their exact relationships in simple supported beams are presented here. These variables show the nature of the problem in a clear way, and provide a rational basis for selecting the connection.
\end{abstract}

CE Database subject headings: Wood structures; Composite beams; Connections; Conceptual design.

\section{Introduction}

Several authors approached the problem of the mixed beam (simple bending) with flexible connections in the 1940s and 1950s (Newmark et al. 1951). From that time and up to the present, many studies have dealt with this issue. The problem has been solved in a global way with the flexibility method (Tommola and Jutila 2001) and even more importantly: the stiffness matrix of the element has also been solved (Amadio 1990; Amadio and Fragiacomo 1993) even using a nonlinear approach for the connection (Salari et al. 1998) (also see the following Ph.D. theses: Norlin 1993; Van der Linden 1999; Fragiacomo 2000; Dias 2005; and Jorge 2005).

The formulation of the Eurocode-5 (CEN 1995, 1996, 2003), to a great extent based on previous investigations, solves the problem in a very precise way with just a simple formulation (see also Ceccotti 1995; Kreuzinger 1995).

From the viewpoint of the process of design, the basic problem lies in determining, a priori, the efficiency of the proposed solution, in relation to its lower and higher bound. This research shows that the efficiency strength is controlled with only one nondimensional parameter. This parameter depends on the stiffness per length unit of the connection, on the mechanical and geometrical characteristics of the two sections to be joined, and on the span. The more important stiffness efficiency is controlled with only two nondimensional variables, one of them being previously mentioned, and the other one the shape factor of the beam section.

Using these nondimensional expressions, a more rational design process of the mixed beam can be performed, where the deviation between the stiffness of the proposed solution, that of the optimum solution, and that of the worst one can directly be controlled.

This way of approaching the problem not only provides a higher control within the design process, but also reveals the basic variables of the problem and their relationship.

\section{Derivation of Differential Equation in Simple Bending}

The first step is to infer the differential equation according to the approach proposed by Newmark et al. (1951); the equation is then tested to assure that it can also be used for the most general case. This is interesting regarding the practical use, as will later be seen in the proposed example showing a gap between the pieces.

The use of the differential equation fulfills the purpose of handling the problem in a symbolic way and not in a numerical mode, as well as approaching the problem in a much more compact solution. In addition, the fundamental physical meaning of the differential equation constants is shown.

The analysis is carried out maintaining the following assumptions:

1. The connection, made in a local or continuous way, is uniform, and so are the geometrical and mechanical characteristics of the pieces (this affects the resolution of the differential equation, but not its layout);

2. The slip of the connection is proportional to the applied sheal connection force; 


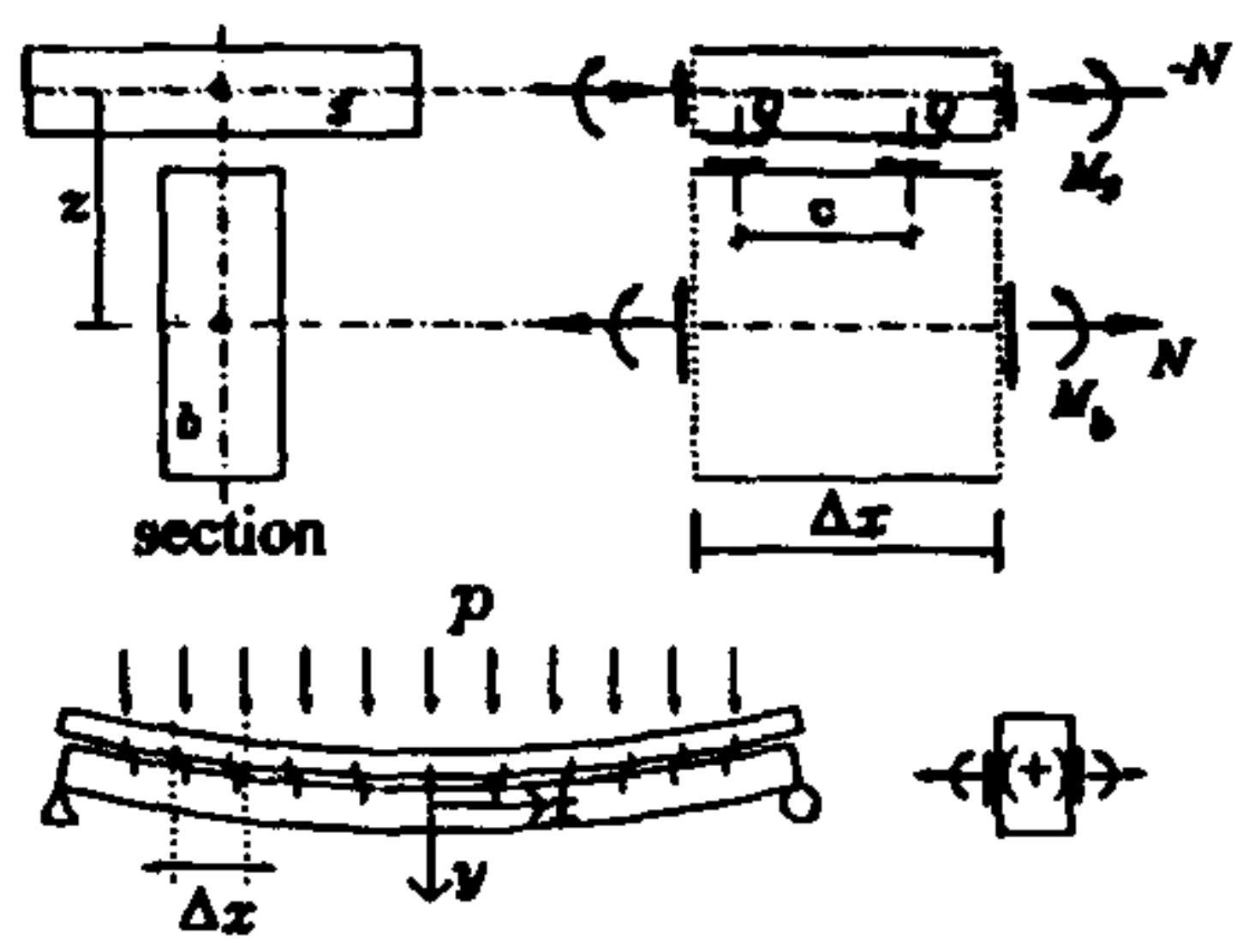

Fig. 1. Type section with gap and sign criterion

3. The Navier-Bernouilli hypothesis used for strength of materials is considered valid inside every piece;

4. The material joining the pieces suffers displacement only due to the shear connection force and it does not resist normal stresses in the direction of the piece;

5. Deformations are small and first-order relations can be used for the strain-stress relationship; and

6. Relative deflection (span/deflection) is small, and therefore the curvature of the two pieces can be considered equal to that of the beam.

A mixed beam composed of two elements, made of two different materials and joined together at a certain distance (see Fig. 1) is analyzed. This layout is sometimes adopted in the mixed wood-concrete structure using tongue and groove planks as permanent formwork and vertical joints (as can later be seen in the example).

The global bending moment, $M$, (see Fig. 1 ) is split up into a pair of forces acting on the gravity center of each piece, $N$, and two bending moments, $M_{s}$ and $M_{b}$, also acting upon the gravity centers mentioned. Therefore

$$
M=N z+M_{s}+M_{b}
$$

The equal descent at both edges of the mixed beam implies a curvature sameness and a rotation sameness; and also using Eq. (1) the following can be established

$$
\chi=\frac{M_{s}}{E_{s} I_{s}}=\frac{M_{b}}{E_{b} I_{b}}=\frac{M-N z}{E_{s} I_{s}+E_{b} I_{b}} \cong-\frac{d^{2} y}{d x^{2}}
$$

where the last equality offers the possibility of directly integrating the elastic equation after determining the equation for the axial stress $N$.

Once the horizontal stresses have been balanced (see Fig. 2) in an infinitesimal length $(d x)$, and taking any of the two parts, the distributed shear connecting force $q$ is obtained

$$
d N=q d x \Rightarrow \frac{d N}{d x}=q
$$

The relation between the shear connecting force in a connector, $Q$, and the distributed shear force, $q$, is immediate (see Figs. 1 and 2)

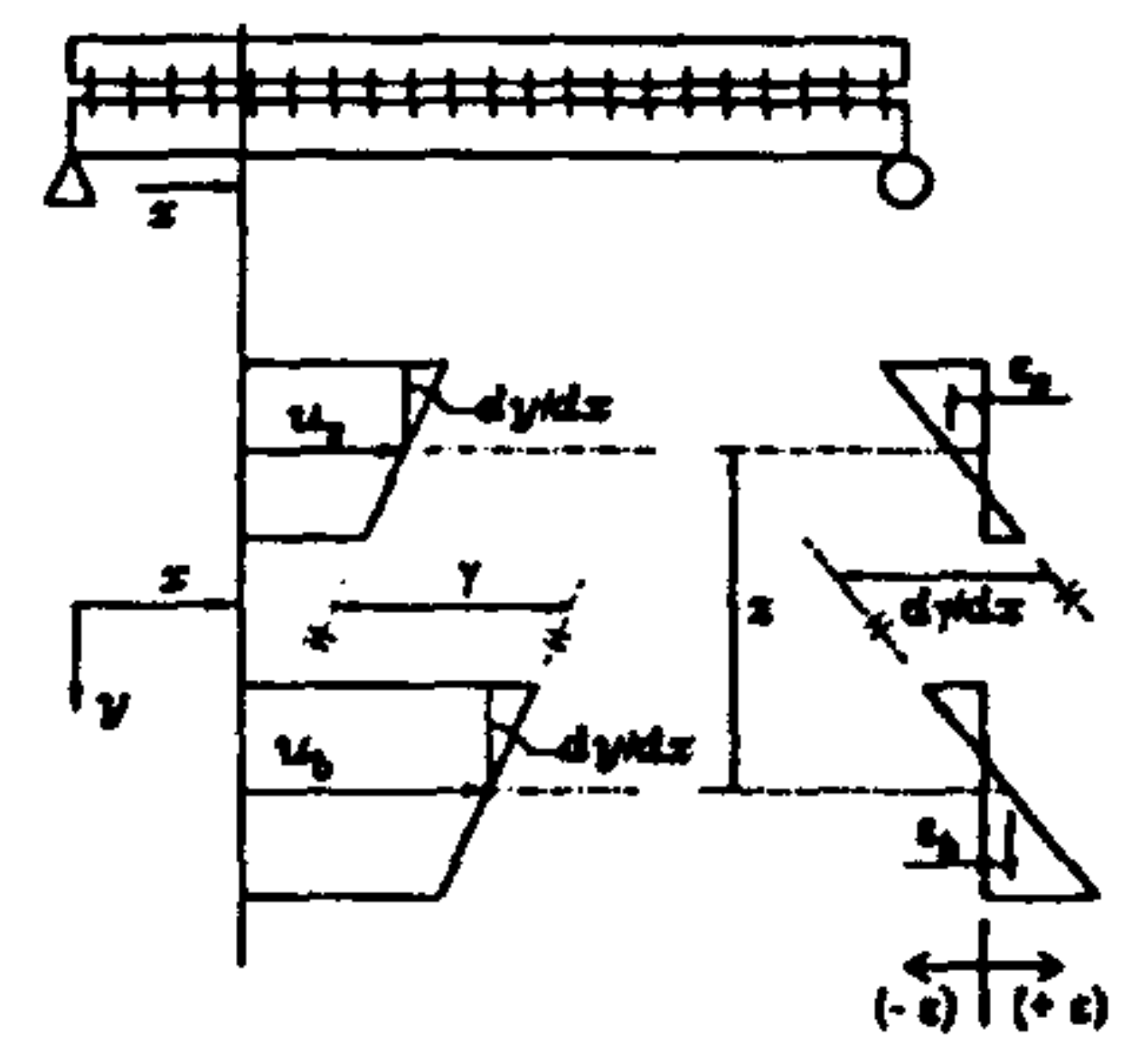

Fig. 3. Slip versus displacements and strains

$$
q=Q / c
$$

Assumption (2) previously mentioned implies the following equation

$$
Q=K_{\text {ser }} \gamma
$$

As can be seen, the stiffness of the connector is not the key parameter; instead, as will be later explained, the key parameter is defined by the unitary stiffness of the connection $\boldsymbol{k}_{\text {unn }}$

$$
k_{\mathrm{um}}=K_{\mathrm{sen}} / c
$$

Fig. 3 shows, for any of the sections, the following functions: $y$ (vertical displacements), $d y / d x$ (rotation), and $\left(u_{b}, u_{s}\right)$ (horizontal displacements). From these functions the differential equation can be approached in a pedagogical manner (see Kreuzinger 1995); and it is easily understandable in the strains diagram $\epsilon$.

The partial connection implies the existence of a slip, $\gamma$, at any beam section, in between the displacement functions of the two parts of the beam $\left(u_{b}, u_{s}\right)$. From Fig. 3 the following can be inferred

$$
\gamma=u_{b}-u_{s}+z \frac{d y}{d x}
$$

The later equation implies a criterion of signs for the $\boldsymbol{\gamma}$ function in accordance with the positive sign of the shear defined in Fig. 1.

The relation between the axial force, $N$, and the connection displacement functions, $\left(u_{b}, u_{z}\right)$, is

$$
\frac{1}{k_{\text {unt }}} \frac{d N}{d x}=\gamma=u_{b}-u_{s}+z \frac{d y}{d x}
$$

The problem has two theoretical bounds with a clear physical meaning.

The term null connection refers to the case in which there is no connection, and therefore $k_{\text {umt }}=0$ and $d N / d x=0$. Hence, there would be two pieces without connection and nonfriction.

The term total connection is used for the state with null slip, $\gamma=0$, which mathematically implies $k_{\text {unt }}=\infty$. This is the case of a continuous piece, or for one with an extremely efficient connection-for example, with the use of very rigid adhesivesgiving the piece a great continuity; therefore, the diagram of strains $\epsilon$ will not show any discontinuity.

In the case of a section with uniform unitary stiffness at the connection ( $k_{\text {un }}=$ constant), derivating Eq. (8), the following can be obtained

$$
\frac{1}{k_{\text {unx }}} \frac{d^{2} N}{d x^{2}}=\frac{d \gamma}{d x}=\frac{d u_{b}}{d x}-\frac{d u_{s}}{d x}+z \frac{d^{2} y}{d x^{2}}=\varepsilon_{b}-\varepsilon_{s}-z X
$$

Inferring from Fig. 3

Fig. 2. Axial force, $N$, versus distributed shear force, $q$ 


$$
\begin{gathered}
\varepsilon_{b}=\frac{N}{E_{b} A_{b}} \\
\varepsilon_{s}=\frac{-N}{E_{s} A_{s}}
\end{gathered}
$$

By substituting Eqs. (2), (10), and (11) in Eq. (9), the differential equation searched is inferred

$$
\frac{1}{k_{\text {unt }}} \frac{d^{2} N}{d x^{2}}=\frac{N}{E_{s} A_{s}}+\frac{N}{E_{b} A_{b}}-\frac{M-N z}{E_{s} I_{s}+E_{b} I_{b}} z
$$

The differential equation obtained coincides with the one obtained by Newmark et al. (1951), but the validity of this equation for the case in which there is a gap between the two pieces should be verified. This situation had not been approached by Newmark.

In order to make the equation easier to operate, some of the variables are grouped together-in the same way Newmark had done-since they have a physical meaning

$$
\frac{1}{\overline{\mathrm{EA}}}=\left(\frac{1}{E_{s} A_{s}}+\frac{1}{E_{b} A_{b}}\right)
$$

where $\overline{\mathrm{EA}}=$ equivalent axial stiffness

$$
\mathrm{EEI}=E_{s} I_{s}+E_{b} I_{b}
$$

(equivalent bending stiffness in the case of null connection)

$$
\mathrm{El}_{\mathrm{eq}}=\Sigma \mathrm{EI}+\overline{\mathrm{EA}} z^{2}
$$

(equivalent bending stiffness in the case of total connection)

and gathering together those terms for a clearer understanding

$$
\begin{gathered}
\lambda=\frac{\mathrm{EI}_{\mathrm{oq}}}{\overline{\mathrm{EA} \Sigma \mathrm{EI}}} \\
\beta=\frac{z}{\Sigma E I}
\end{gathered}
$$

the differential equation is stated then as

$$
\frac{d^{2} N(x)}{d x^{2}}-k_{\text {unt }} \lambda N(x)=-k_{\text {unt }} \beta M(x)
$$

\section{Derivation of Efficiency Parameter of Section Shape, $\alpha$}

Before solving this equation, the value of the axial force for the optimum case without slip (total connection), $N_{\text {OPT }}(x)$, needs to be determined. For this purpose, the only condition is to set the slip function, $\gamma$, as a null value. According to this and using Eqs. (9) and (12)-(14) the following equation is obtained

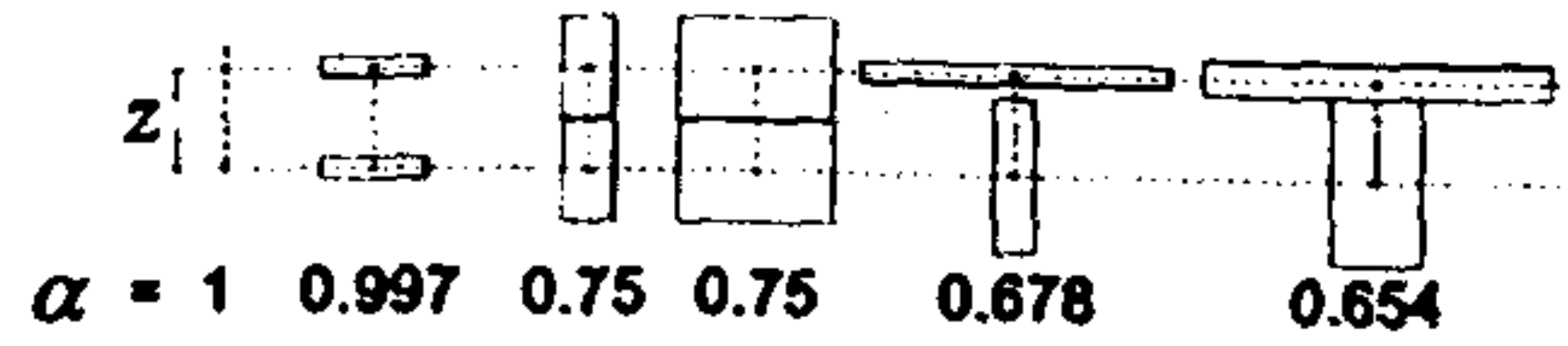

Fig. 4. Examples of $\alpha$ value in different sections

$$
\varepsilon_{b}-\varepsilon_{s}+z \frac{d^{2} y}{d x^{2}}=0=\frac{N_{\mathrm{OPT}}(x)}{\overline{\mathrm{EA}}}-z \frac{M-z N_{\mathrm{OPT}}(x)}{\Sigma \mathrm{EI}}
$$

and substituting Eqs. (16) and (17) the searched value is obtained

$$
N_{\mathrm{OPT}}(x)=M(x) \frac{z \overline{\mathrm{EA}}}{\mathrm{EI}_{\mathrm{eq}}}=M(x) \frac{\beta}{\lambda}
$$

where the axial force is proportional to the bending moment.

The equality in the middle of Eq. (20) is the traditional one for two piece beams formed of different materials, where $\mathrm{EI}_{\text {eq }}=$ stiffness equivalent to bending and $2 \overline{\mathrm{EA}}=$ static moment for each piece, weighting each one by its corresponding elasticity module in relation to the geometric center of the whole mixed section.

Defining a new nondimensional parameter $\alpha$ becomes of great interest

$$
N_{\mathrm{OPT}}(x)=M(x) \frac{\beta z}{\lambda z} \Rightarrow N_{\mathrm{OPT}}(x)=\frac{M(x)}{z} \alpha
$$

where

$$
\alpha=\frac{\beta}{\lambda} z=\frac{z^{2} \overline{\mathrm{EA}}}{\Sigma \mathrm{EI}+z^{2} \overline{\mathrm{EA}}}=\frac{z^{2} \overline{\mathrm{EA}}}{\mathrm{EI}_{\text {eq }}}=\frac{\mathrm{EI}_{\mathrm{oq}}-\Sigma \mathrm{EI}}{\mathrm{EI}_{\text {oq }}}
$$

This parameter, $\alpha$, measures the efficiency of the section shape weighted by each Young module or, in other words, the effectiveness of the total homogeneous section. Its domain is: $0<\alpha<1$. The value of $\alpha$ when $E=E_{s}=E_{b}$ is of great interest and is stated

$$
\alpha=\frac{z^{2} \bar{A}}{\Sigma I+z^{2} \bar{A}}
$$

That is (see Fig. 4), in any truss beam or sandwich structure optimum values are reached. When both pieces are identical and there is no separation, the value of alpha is always 0.75 . In the common T-shaped beam, with floor sections made of wood or wood and concrete, $\alpha$ valués are all $0.65<\alpha<0.8$.

\section{Solution of Differential Equation in Simple Bending}

The general solution of the differential equation, solved with MAPLE, and expressed according to the optimum axial force, $N_{0}(x)$, is

$$
N(x)=\underbrace{C_{1} e^{\phi x}+C_{2} e^{-\phi x}}_{\text {general solution of mermugencous equation }}-\underbrace{\frac{1}{2} \phi\left[e^{\phi x} \int e^{-\phi x} N_{\mathrm{OPT}}(x) d x-e^{-\phi x} \int e^{\phi x} N_{\mathrm{OPT}}(x) d x\right]}_{\text {punticular solution }}
$$




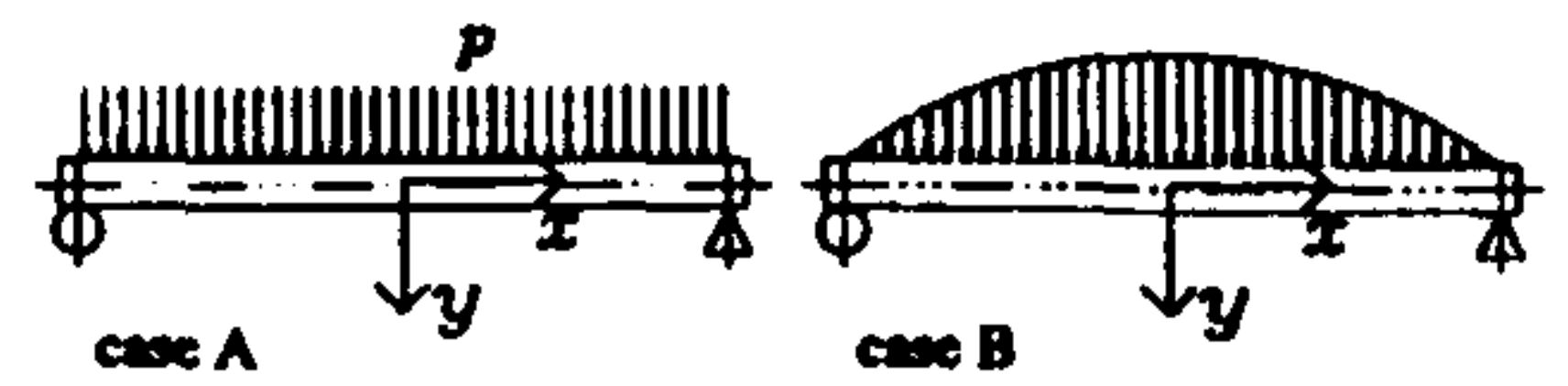

Fig. 5. Load cases ( $A=$ constant load; $B=$ cosine-shaped load)

where

$$
\phi=+\sqrt{\left(k_{\mathrm{unt}} \lambda\right)}
$$

where $C_{1}$ and $C_{2}=$ constants that depend on the boundary conditions.

Interestingly, the first two monomials belong to the general solution of the homogeneous equation, and the third one belongs to a particular solution which, in this case, depends on the optimum axial force, $N_{\text {OPT }}(x)$.

\section{Case Analysis for Simple Supported Beam}

Two specific cases (see Fig. 5) are analyzed: the supported beam with a uniform load, the most common case, and the supported beam with cosine-shaped load showing the same value at the maximum moments; the model is used by Eurocode 5.

The value of the bending moment is

$$
\begin{aligned}
& \text { Case } A \Rightarrow M(x)=-\frac{1}{2} p x^{2}+\frac{1}{8} p L^{2} \\
& \text { Case } B \Rightarrow M(x)=\frac{1}{8} p L^{2} \cos \left(\frac{\pi x}{L}\right)
\end{aligned}
$$

The use of the cosine-shaped load remarkably simplifies the expressions with more than reasonable precision. The case of constant load has been solved just for comparing and measuring the error of approximation.

Although the analysis is reduced to these two cases, the consequences will be much more general.

\section{Design Parameters in Strength}

Once the differential equation has been solved depending on the axial force [Eq. (24)], the strength efficiency, $\mu(s)$, is measured with

$$
\mu(s)=\frac{N_{K u n l, \max }}{N_{\text {OPT,max }}}
$$

where $N_{\text {Kunt.max }}=$ maximum axial force in the case of slip; and $N_{\text {OPT }, \max }=$ maximum axial force in the case of total connection [see Eq. (20)].

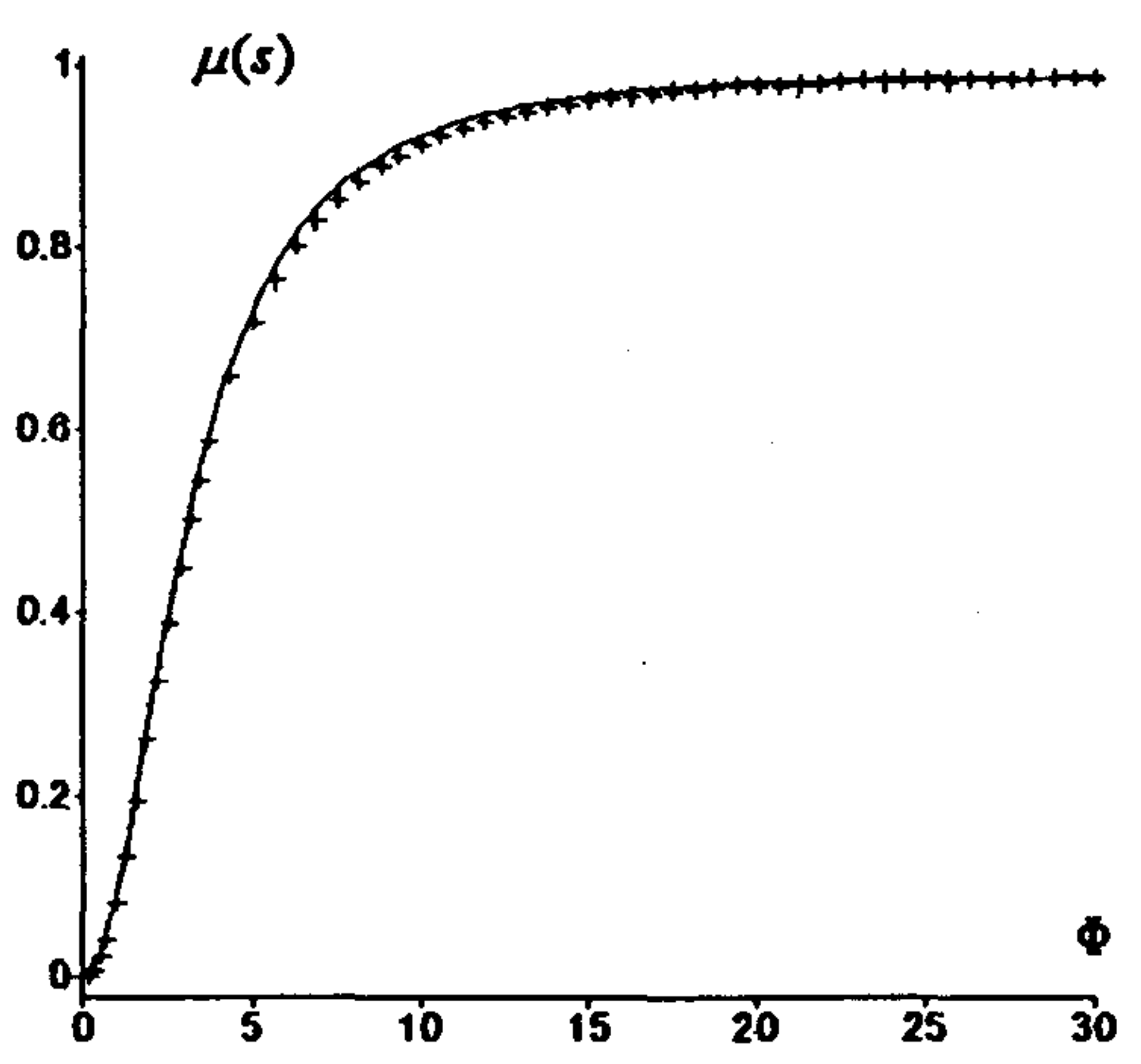

Fig. 6. Strength efficiency $\mu(s)$ versus $\Phi$ (cross $\rightarrow$ cosine-shaped load; continuous $\rightarrow$ constant load)

In Case $A$, with a uniform load (Fig. 5), the axial force is [with $\phi$ defined in Eq. (25)]

$$
N(x)=\frac{\left(\frac{e^{-(1 / 2) \phi L}\left(e^{\phi x}+e^{-\phi x}\right)}{e^{-\phi L}+1}-1\right) \beta p}{\lambda^{2} k_{\text {min }}}+N_{\text {OPT }}(x)
$$

and the value of the strength efficiency changing the variable

$$
\Phi=\phi L=+\sqrt{\left(k_{\text {umi }} \lambda\right) L}
$$

the results are defined by just one design parameter, $\Phi$

$$
\mu(s)=\frac{8\left(\frac{2 e^{-\Phi / 2}}{e^{-\Phi}+1}-1\right)}{\Phi^{2}}+1
$$

In Case $B$ with cosine-shaped load (see Fig. 5), the axial force is

$$
N(x)=\frac{N_{\mathrm{OPT}}(x)}{1+\left(\frac{\pi}{\Phi}\right)^{2}}
$$

That is, it is proportional to that of the axial force with total connection, $N_{\mathrm{OPT}}(x)$; and the value of the strength efficiency, $\mu(s)$, is (see Fig. 6)

$$
\mu(s)=\frac{1}{1+\left(\frac{\pi}{\Phi}\right)^{2}}
$$

The cosine-shaped load greatly simplifies the problem with a more than excellent approximation. Drawing both equations, a clear image of the difference between both cases can be seen, and even more, the nature of the problem is shown.

Therefore, the increase of $\Phi$ means an efficiency improvement in a more or less linear way until $\Phi \approx \leqslant 4$, point which can be called $\Phi_{\text {LOWER. }}$ Up to $4 \approx \leqslant \Phi \approx \leqslant 10$ the improvement is barely significant, and with $\Phi \approx \geqslant 10$, the point which can be called $\Phi_{\text {UPPER, }}$ the stiffness of the connection has to be increased in an extreme way with practically no advancement. The values of these bounds could change for other kinds of problems (different from the simple supported beam), but they will always exist and they can easily be determined numerically in every case. 


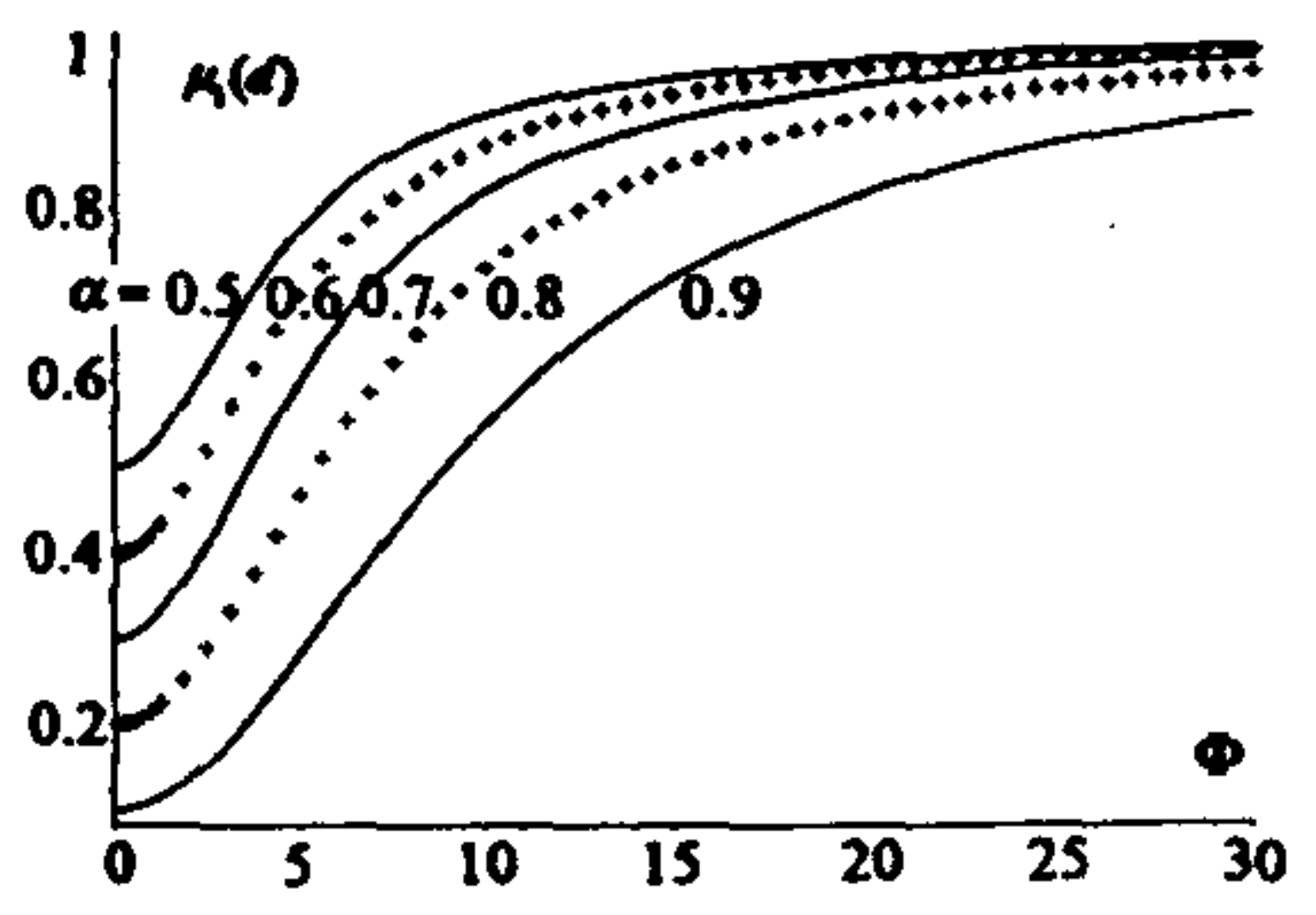

Fig. 7. Stiffness efficiency $\mu_{1}(d)$ versus $\Phi$ and $\alpha$

By using the expressions of stress efficiency, for a specific case, the $k_{\text {unt }}$ of the connection can be directly determined, for a reasonable strength performance, without performing iterative processes. Later, design calculations should be carried out to find out if it is constructively possible or feasible to reach the value mentioned with the usual constructive solutions.

\section{Design Parameters in Stiffness}

The deflection function of the beam, $\delta(x)$, has been solved by the double integration of the curvature $\chi(x)$ [see Eq. (2)] regarding the well known relation

$$
\frac{d^{2} \delta(x)}{d x^{2}}=-\chi(x)
$$

The stiffness efficiency of the solution, $\mu_{1}(d)$, is measured as

$$
\mu_{1}(d)=\frac{\delta_{\text {OPT,max }}}{\delta_{\text {Kunt,max }}}
$$

where $\delta_{\text {OPT,max }}=$ maximum deflection with total connection and where $\delta_{\text {Kunt, } \max }=$ maximum deflection in the case of slip at the connection.

In Case $A$, with a continuous load (see Fig. 5), the value of $\mu(d)$ is

$$
\mu_{1}(d)=\frac{1}{1-\frac{768}{5} \frac{\alpha e^{\Phi / 2}}{\left(1+e^{\Phi}\right)(-1+\alpha) \Phi^{4}}-\frac{48}{5} \frac{\alpha\left(\Phi^{2}-8\right)}{(-1+\alpha) \Phi^{4}}}
$$

In Case $B$, with a cosine-shaped load (see Fig. 5), the value of $\mu(d)$, is

$$
\mu_{1}(d)=\frac{\left(\Phi^{2}+\pi^{2}\right)(1-\alpha)}{\pi^{2}+(1-\alpha) \Phi^{2}}
$$

The cosine-shaped load simplifies the problem once again in a remarkable way and also, with an excellent approximation, as can be checked by comparing both equations.

Due to the similarity of both Cases $A$ and $B$, only the solution with a cosine-shaped load, the simplest one (see Fig. 7) is drawn.

Once these relationships have been established, the limit values of the equivalent inertia can be obtained for both cases: that of the beam with null connection and with total connection; in short, the bounds of the problems are set.

The equivalent inertia of the beam in the case of total connection, based on the values obtained from the specific cases, is: $E I_{e q}=\Sigma E I+\overline{E A} z^{2}$ (as previously advanced), and in the case of no connection the stiffness is obviously $\mathrm{ZEI}$.

To define the total deflection limits, $\Delta \delta_{\max }$ is stated as the relation between the maximum deflection with null connection

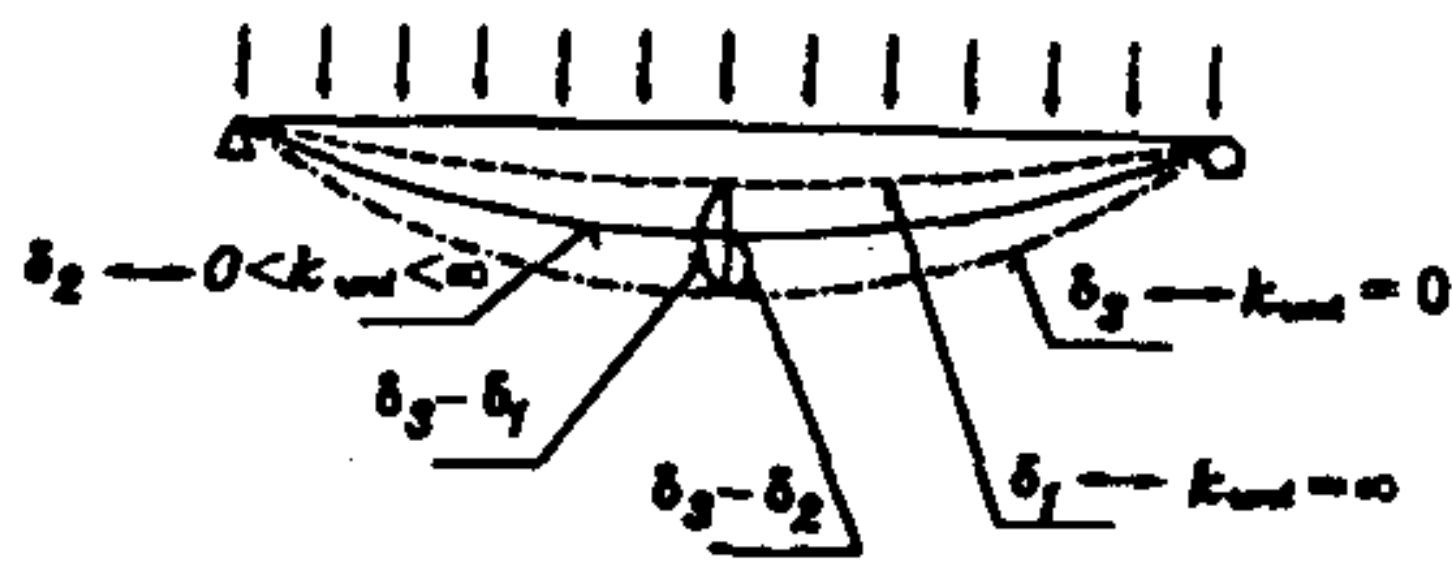

Fig. 8. Alternative definition of stiffness efficiency, $\mu_{2}(d)$

$\delta_{\text {Kunte=.max }}$ and the deflection with total connection $\left(\delta_{O P T, \text { max }}\right) ;$ and as can be verified from the use of the previous expressions, the following is obtained

$$
\Delta \delta_{\max }=\frac{\delta_{\text {Kunt }=0, \max }}{\delta_{\text {OPT,max }}}=\frac{1}{1-\alpha}
$$

That is, the bounds of the stiffness benefits are defined by the shape of the homogeneous section. For usual alpha values, 0.6 $\leqslant \alpha \leqslant 0.8$, the maximum benefit is $2.5 \leqslant \Delta_{\max } \leqslant 5$.

Another way of defining the stiffness efficiency, with great conceptual interest, is also possible (see Fig. 8)

$$
\mu_{2}(d)=\frac{\delta_{3}-\delta_{2}}{\delta_{3}-\delta_{1}}
$$

The denominator, $\left(\delta_{3}-\delta_{1}\right)$, is the greatest reduction of the deflection obtainable with total connection; and the numerator, $\left(\delta_{3}\right.$ $\left.-\delta_{2}\right)$, is the feasible one with a partial connection. Using Eqs. (35), (37), and (38), it is proved that

$$
\mu_{2}(d)=\frac{1}{1+\frac{\pi^{2}}{\Phi^{2}}}=\mu(s)
$$

This definition shows the notable importance of the nondimensional parameter $\Phi$, but it is useless for comparing beams with different section shapes.

Finally, to complete the relation between the strength and stiffness efficiency, using Eqs. (33) and (37), the following can be shown

$$
\mu_{1}(d)=\frac{1-\alpha}{1-\mu(s) \alpha}
$$

\section{Design Example}

A wood-concrete mixed beam, represented in Fig. 9, is designed (assuming that the wood beam is initially shored).

The following data are considered: elasticity modules of $E_{s}=27$ and $E_{b}=9$, both in $\mathrm{kN} / \mathrm{mm}^{2}$; a permanent load of $4 \mathrm{kN} / \mathrm{m}^{2}$; and a variable one of $3 \mathrm{kN} / \mathrm{m}^{2}$. Therefore, the maximum calculated bending moment is: $M_{d, \max }=0.6(1.35 .4$ $+1.5 \cdot 3) 6^{2} / 8=26.73 \mathrm{kN} \mathrm{m}$.

Considering a European C-18 strength class for the wooden beam, the tensile and bending strengths are as follows

$$
\begin{gathered}
f_{1, d}=11 \cdot 0.8 / 1.3=6.77 ; \\
f_{m, d}=18 \cdot 0.8 / 1.3=11.08\left(\text { both in } \mathrm{N} / \mathrm{mm}^{2}\right)
\end{gathered}
$$

Section parameters are

$$
\begin{aligned}
\overline{\mathrm{EA}} & =238,235 \mathrm{kN}[\sec \mathrm{Eq} .(13)] \\
\sum \mathrm{El} & =1,926.5 \mathrm{kN} \mathrm{m}^{2}[\sec \mathrm{Eq} .(14)]
\end{aligned}
$$



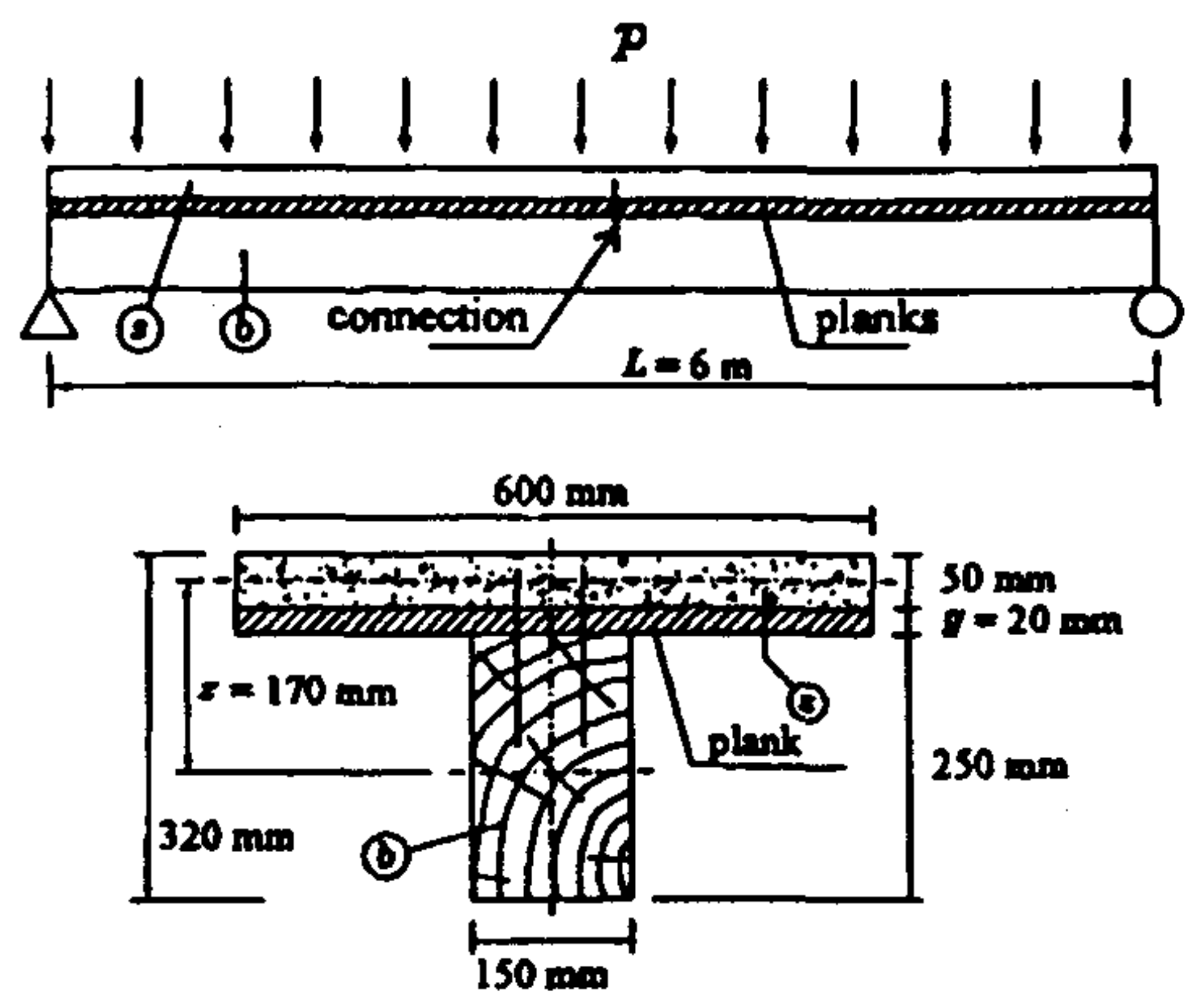

Fig. 9. Design example

$$
\begin{gathered}
\mathrm{EI}_{\mathrm{eq}}=8,811.5 \mathrm{kN} \mathrm{m}^{2} \text { [see Eq.(15)] } \\
\alpha=0.7814 \text { [see Eq.(22)] }
\end{gathered}
$$

Regarding the strength efficiency, the following can be established

$$
\begin{gathered}
N=\mu(s) \alpha M_{d} / z=122.86 \mu(s) \mathrm{kN} \text { [see Eqs.(28)and(21)] } \\
M_{b}=\frac{E_{b} I_{b}}{\sum \mathrm{EI}}\left(M_{d}-z N\right)=24.39-19.06 \mu(s) \mathrm{kN} \mathrm{m} \text { [see Eq.(2)] }
\end{gathered}
$$

A bending-axial interaction according to Eurocode 5 is considered as

$$
\frac{\sigma_{t, d}}{f_{t, d}}+\frac{\sigma_{m, d}}{f_{m, d}} \leqslant 1
$$

and substituting the corresponding values in this last equation (wood strength is considered up to $95 \%$, keeping it as a margin regarding aspects not being analyzed for the moment)

$$
\frac{122,860 \mu(s)}{150 \cdot 250 \cdot 6.77}+\frac{6,000[24.39-19.06 \mu(s)]}{150 \cdot 250 \cdot 0.25 \cdot 11.08}=0.95
$$

from where an efficiency of at least $\mu(s)=0.7436$ is obtained.

From Eq. (33) the value of the nondimensional parameter $\Phi$ is inferred

$$
\Phi^{2}=\pi^{2} \frac{\mu(s)}{1-\mu(s)}=28.62
$$

and combining Eqs. (30), (16), and (22) the minimum unitary stiffness the joint system needs to have is obtained

$$
k_{\text {unt }}=\Phi^{2}(1-\alpha) \frac{\overline{E A}}{l^{2}}=41.40 \mathrm{~N} / \mathrm{mm}^{2}
$$

- To finish, the next step is to check that the constructive solution chosen is feasible for this case.

Vertical dowels are used and according to Gelfi et al. (2002) (using a simplified relation for the qualities of common use materials) the stiffness of the connector is

$$
K_{\text {ser }}=\frac{124,000 d}{\left(4.34+\frac{g}{d}\right)^{3}}
$$

with $K_{\text {ser }}$ in $\mathrm{N} / \mathrm{mm} ; d$ and $g$ in $\mathrm{mm}$. For dowels of $d=10,12$, and $14 \mathrm{~mm}, g=20 \mathrm{~mm}$, and according to Eurocode 5, the following
Table 1. Required Values of $k_{u n t}$ in $N / \mathrm{mm}^{2}$

\begin{tabular}{lccccc}
\hline & \multicolumn{5}{c}{$\begin{array}{c}c \\
(\mathrm{~mm})\end{array}$} \\
\cline { 2 - 6 }$(\mathrm{mm})$ & 80 & 100 & 120 & 150 & 200 \\
\hline 10 & 40.55 & 32.44 & 27.03 & 21.63 & 16.22 \\
12 & - & 45.77 & 38.14 & 30.52 & 22.89 \\
14 & - & - & 50.24 & 40.19 & 30.15 \\
\hline
\end{tabular}

unitary stiffness of connection $k_{\text {unt }}$, for a different $c$ separation between the connections, is obtained (see Table 1 ) [since it is a verification of the ultimate limit state, (ULS) $K_{\text {ser, UL } s}=2 K_{\text {ser }} / 3$ ].

Hence, the problem is feasible for this type of joint and a joint system with the appropriate efficiency can also be chosen for each case. It should be forgotten that there are some joining systems that are not feasible for certain spans or loads, and this is not always known by the designer.

Other types of solutions can be suggested, but this example has shown the use of nondimensional variables in the connection design.

The proposed solution has a capacity of calculated bending of $M_{d}=26.73 \mathrm{kN} \mathrm{m}$, that is, a strength efficiency of $\mu(s)=0.74$, associated with a $\Phi=5.35$ parameter with $k_{\text {um }}=41.40 \mathrm{~N} / \mathrm{mm}^{2}$.

Looking now at a case, for example, with two connectors per connection, and relatively close to each other, $k_{\mathrm{um}}=80 \mathrm{~N} / \mathrm{mm}^{2}$ has been obtained. With this rigidity $\Phi=7.44$ is obtained, and an axial efficiency of $\mu(s)=0.849$, which does not mean a great increase (see Fig. 6). That is, the key point is that each joining system has an upper bound from which the solution is not to place a greater number of connectors but to change to a more rigid connection (a higher $k_{\text {un }}$ value).

In a similar way, the design could be carried out by strength efficiency $\mu(d)$ although, as has already been seen, stiffness efficiency and strength efficiency are totally related.

\section{Assumptions and Limits of Application}

It is worth revising the practical limits of the assumptions on which this research has been developed, and by doing so to again state the true aim of this proposal.

First, the model assumes a linear load-deformation relationship at the connection. Different tests have shown that this is a reasonable assumption under service loads in many cases, as has already been stated (Vander Linden 1999). Nevertheless, it is also clear that this very important assumption cannot be drawn for any connectors; and most importantly, the assumption is neither valid for a close to ultimate load nor for a nonsimplified (nonlinear) long-term analysis.

Second, a linear constitutive model is also assumed for the two pieces of the beam. Nonlinearity, in general, can only be considered with this model through the use of equivalent linear values, as it is presently set at Eurocode 5. For a recent study on longterm behavior of timber-concrete composite beams see Amadio (2001), Fragiacomo (2000, 2005, 2006); Fragiacomo and Ceccotti (2006), and Kuhlmann and Schänzlin (2001, 2004).

Nevertheless, it has to be emphasized that in spite of the consideration of linear constitutive models for the joint and for the two pieces to be bound, the global behavior of the beam is not linear in relation to the change in the stiffness of the connection. This is the main aspect which allows establishing a particular debate around the connection selection. 
Third, the gap considered in the type section analyzed shows a major conceptual interest, as it unifies the treatment of problems normally treated separately, even though it is important to keep in mind, when relating it to practical terms, that the separation of pieces is not compatible with all types of joining systems. The case shown in the example has a major practical interest, but the vertical dowels do not admit separations greater than the ones established there, as the deformation by bending could imply small $k_{\text {um }}$ values or even be insignificant. Great separations are normally associated with the sandwich case, in which the foam works both as a thermal insulator and as connector of great stiffness. In any case, the important data in order to reach an efficient connection system, within the assumption made, is simply $\Phi$, intervening the connecting system through $k_{\text {unv }}$.

In other words, the simplifications assumed in the model become relevant when the main goal of the research is that of rationalizing, with an analytical base, the selection process of the connection which is definitely not a minor aspect in the process of design of the composite beams with flexible connections.

\section{Conclusions}

The work shows how the nonlinearity of the problem implies the existence of two bounds, $\Phi_{\text {LOWER }}$ and $\Phi_{\text {UPPER, }}$, defined by only one nondimensional variable. This allows choosing in advance, for a given problem, the value of the stiffness per length unit of the connection in a way that reasonable strength efficiency (and then also stiffness efficiency) is kept. The variable $\Phi$ depends on the stiffness per length unit of the connection, on the span, and on the geometrical and mechanical characteristics of the sections to be joined.

The stiffness efficiency also depends on two nondimensional variables, $\Phi$, and another nondimensional parameters $\alpha$. The latter depends only on the shape of the total homogeneous section.

The limits of the section stiffness are defined by parameter $\alpha$. The higher the alpha value, the higher the stiffness, even though it is more difficult to reach the connection.

The nondimensional processing of the variables allows them to be reduced to a minimum number, and it also facilitates the understanding of the nature of a complex problem, which nevertheless can be used in a relatively easy and clear way.

\section{Acknowledgments}

The results presented here were developed within the research project: "Wood-concrete floors: A protocol for refurbishment." This project was carried out with the financial support of the Spanish Government within the I+D-2004 National Research Program.

\section{Notation}

The following symbols are used in this paper:

$A=$ cross-sectional areas;

$b$ = lower piece (beam);

$c=$ separation between local connections;

$d=$ diameter of dowel (as subindex, calculation value);

$E=$ clastic Young's module;

$f_{m, d}=$ bending strength regarding bending moment;

$f_{t, d}=$ stress strength regarding axial force;

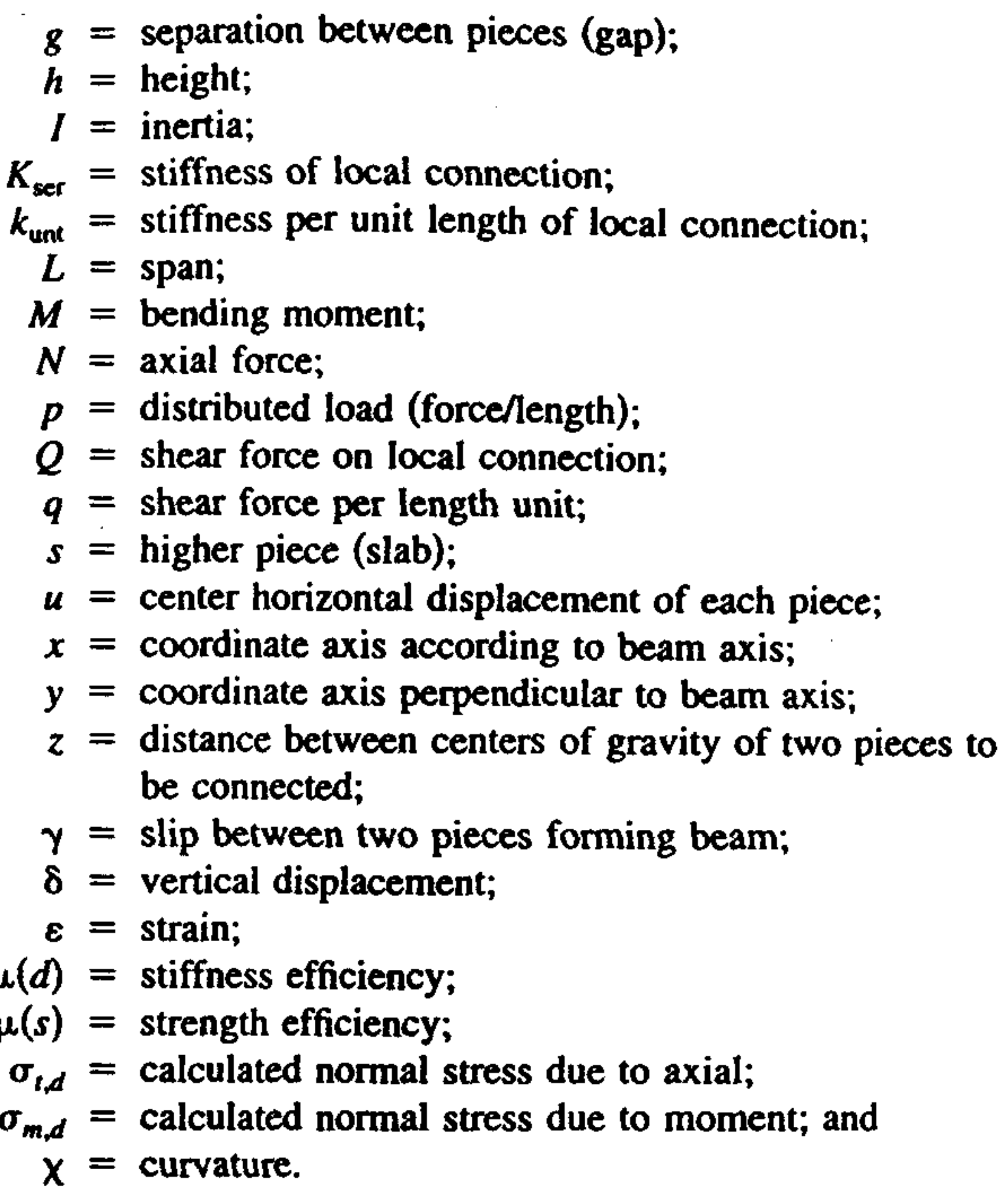

\section{References}

Amadio, C. (1990). "Elementi finite per lo studio flessionale elatico di travi composte di connessioni deformabili." Constr. Metallique, 6, 365-374.

Amadio, C. (2001). "Influence of theological phenomena in timberconcrete composite beams." Proc., IABSE Conf. on Innovative Wooden Structures and Bridge, Lathi, Finland, 525-529.

Amadio, C., and Fragiacomo, M. (1993). "A finite element model for the study of creep and shrinkage effects in composite beams with deformable shear connections." Constr. Metallique, 4 213-228.

Ceccotti, A. (1995). "Lecture E13: Timber-concrete composite structures." STEP 2. Timber engineering. Centrum Hout, The Netherlands, E13/1-12.

Comité Europken de Normalisation (CEN). (1995). "Design of timber structures-Part 1-1: General rules and rules for buildings." Eurocode 5, ENV 1995-1-1, Brussets, Belgium.

Comite Europten de Normalisation (CEN). (1996). "Design of timber structures-Part 2: Bridges." Eurocode 5, ENV 1995-2, Brussels, Belgium.

Comite Européen de Normalisation (CEN). (2003). "Design of timber structures-Part 1-1: General rules and rules for buildings." Eurocode 5, prENV 1995-1-1, Brussels, Belgium.

Dias, A. (2005). "Mechanical behaviour of timber-concrete joints." Ph.D. thesis, Technische Univ. Delft, The Netheriands.

Fragiacomo, M. (2000). "Long-term behaviour of timber-concrete composite beams." Ph.D. thesis, Univ. of Trieste, Trieste, Italy, in Italian.

Fragiacomo, M. (2005). “A finite element model for long-term analysis of timber-concrete composite beams." Struct. Eng. Mech., 20(2), 173-189.

Fragiacomo, M. (2006). "Long-term behavior of timber-concrete composite beams. II: Numerical analysis and simplified evaluation." J. Siruct. Eng., 132(1), 23-33.

Fragiacomo, M., and Ceccotti, A. (2006). "Long-term behavior of timberconcrete composite beams. I: Finite element modeling and validation." J. Struct. Eng., 132(1), 13-22.

Gelfi, P., Giuriani, E., and Marini, A. (2002). "Stud shear connection design for composite concrete slab and wood beams." J. Siruct. Eng., 128(12), 1544-1550. 
Tange, L. (2(x)5). "Timber-concrete composite structures using LWAC." Ph.D. thesis. Coimbra Univ.. Portugal (in Portuguese).

Kuhlmann. U., and Schänzlin. J. (2(0)1). "Composite of vertically timber decks and concrete." Proc.. IABSE Conf. on Innovative Woroden Structures and Bridge, Lathi. Finland, 507-512.

Kuhlmann, U., and Schänzlin, J. (20(4)). "Composite of vertically timber decks and concrete." Proc., of $8 \mathrm{th}$ World Conf. of Timber Engineering, Lathi, Finland, 313-318.

Kreuzinger, H. (1995). “Lecture B II: Mechanically jointed beams and colums." STEP I. Timber engineering, Centrum Hout, The Netherlands. $B 11 / 1-8$.

MAPLE v9.0. "Mathematical software." Maplesoft.

Newmark, N. M.. Siess, C. P., and Viest, I. M. (1951). "Test and analysis of composite beams with incomplete interaction." Proc. Soc. Exp. Siress Anal., 9(1), 75-92.

Norlin, B. (1993). "Two-layered composite beams with nonlinear connectors and geometry-test and theory." Ph.D. thesis, Royal Institute of Technology, Stockholm, Sweden.

Salari, R. M., Spacone, E., Shing, P. B., and Frangopol, D. (1998). "Nonlinear analysis of composite beams with deformable shear connectors." J. Struct. Eng., 124(10), 1148-1158.

Tommola, J., and Jutila, A. (2001). "Analysis of wood-concrete composite girder with discrete shear connectors." Proc., IABSE Conf. on Innovative Wooden Structures and Bridges, Lathi, Finland, 293-296.

Van der Linden, M. (1999). "Timber-concrete composite floor systems." Ph.D thesis, Technische Univ., Delft, The Netherlands. 